\title{
La responsabilidad oceánica de Chile: ideas para una política exterior marítima vinculada al desarrollo sostenible
}

\author{
Lucas Pavez Rosales
}

Recibido: 08/02/2020.

\author{
Ipavezrosales@gmail.com \\ Pontificia Universidad Católica de Valparaíso, Chile
}

Aceptado: 05/10/2020

\begin{abstract}
Resumen: Esta investigación presenta, como su objetivo general, proponer el concepto de "responsabilidad oceánica de Chile" y su aplicación en la gestión de las Áreas Marinas Protegidas como impulsor de una política exterior marítima que maximice los intereses nacionales desde su vinculación al desarrollo sostenible. Se expone la creación y desarrollo del concepto de responsabilidad oceánica como una propuesta innovadora pero arraigada en una historia de contribuciones nacionales en la materia. Se busca realizar un aporte con una herramienta que sirva de principio activo para la promoción de Chile como un referente oceánico, compatibilizando la economía, la conservación ambiental, los intereses marítimos y el potenciamiento del desarrollo nacional.
\end{abstract}

Palabras claves: responsabilidad oceánica - política exterior marítima - áreas marinas protegidas - desarrollo sostenible

* Cómo citar este artículo: Pavez Rosales, L. (2020). La responsabilidad oceánica de Chile: ideas para una política exterior marítima vinculada al desarrollo sostenible. Relaciones Internacionales, 29(59), 107. https://doi.org/10.24215/23142766e107

Editor: Juan Alberto Rial, Instituto de Relaciones Internacionales Facultad de Ciencias Jurídicas y Sociales (Universidad Nacional de La Plata)
Entidad editora: Relaciones

Internacionales, es una publicación del Instituto de Relaciones Internacionales (Facultad de Ciencias Jurídicas y Sociales (Universidad Nacional de La Plata Argentina)

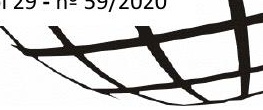




\title{
Chile's Oceanic Responsibility: ideas for a sustainable development related maritime foreign policy
}

\section{Lucas Pavez Rosales ${ }^{1}$}

\begin{abstract}
This research has the following general objective: putting forward the concept "Chile's oceanic responsibility" and its implementation on the management of marine protected areas to strengthen a marine foreign policy capable of boosting national interests from its connection to sustainable development. This paper presents the creation and development of the concept of Oceanic Responsibility as an innovative proposal based on a long history of national contributions to the matter. It seeks to make a contribution by using an active principle to promote Chile as an oceanic reference, harmonizing economy, environmental conservation, maritime interests and national development.
\end{abstract}

Key words: oceanic responsibility - maritime foreign policy - marine protected areas sustainable development

1 Tercer Secretario del Servicio Exterior, Ministerio de Relaciones Exteriores de Chile. Magíster en Relaciones Internacionales de la Universidad Nacional de La Plata, Argentina. Profesor de Historia, Geografía y Ciencias Sociales, y Licenciado en Historia mención Ciencia Política, de la Pontificia Universidad Católica de Valparaíso, Chile.

Las palabras vertidas en este texto son de exclusiva responsabilidad de quien las escribe y no representan necesariamente los lineamientos del Ministerio de Relaciones Exteriores de Chile. 


\section{Introducción}

El estudio de la política exterior en el marco de las relaciones internacionales debe tener siempre presente su relación con la política interna de un país. En ese sentido, Jaguaribe postulaba que la construcción de la política exterior es "una ecuación de optimización, en el ámbito internacional, de los principales intereses de un país, teniendo en cuenta sus condiciones internas y externas y los medios de acción de que dispone" (1974, p. 104) donde el modelo de inserción global debe ser una selección objetiva. Ello se complementa con lo que Duroselle indicaba, que "no existe ningún acto de política extranjera que no tenga un aspecto de política interna" (2000, p. 53) desde el ejemplo de cómo una alianza internacional enfrenta facciones políticas internas, y que estas decisiones pueden producir efectos externos con costos internos o ser tomadas con cálculo mixto para producir efecto al exterior e interior.

La política exterior es una proyección de la política interna de un país, son dos caras de una misma moneda, antes que polos diferentes. La política interna y la política exterior están en interrelación y abundan los mecanismos de articulación entre ambas, en un proceso sistémico donde la política exterior de un Estado viene condicionada por la situación interna y por la política que al interior de ese Estado se desarrolle. Al mismo tiempo, la política interna va a estar influida y orientada por las acciones llevadas a cabo hacia el exterior y las repercusiones que ellas traigan.

La política exterior de Chile no está exenta de esto y, desde la década de 1990, ha tomado una decisión estratégica para alcanzar el crecimiento económico y bienestar social (aunque la desigualdad sigue siendo una de las trabas para el desarrollo), la cual ha trazado un camino constante en materia de política exterior, independiente de la coalición gobernante. Así, la inserción de Chile en el mundo globalizado ha estado fundada en su autopercepción como "un país mediano, no periférico, integrado al mundo y a la región, exitoso económicamente y estable desde el punto de vista político-institucional" (Colacrai \& Lorenzini, 2005, p. 47).

Su inserción se caracteriza por la apertura económica mediante la suscripción de acuerdos bilaterales de libre comercio y la promoción del valor que tiene el multilateralismo como su premisa, a partir de la convicción de que la construcción de un mundo con reglas claras y construidas entre todos es la mejor forma para dar predictibilidad internacional, lo cual favorece a los países como Chile, que, sin ser rule-makers, buscan maximizar su voz y proponer ideas e iniciativas desde el marco de igualdad que otorga el multilateralismo.

Dentro de este marco, hay temas que destacan por ser prioritarios para Chile, como la defensa y promoción de los derechos humabnos, la integración regional, temas antárticos y, por supuesto, los temas oceánicos. La razón es que la relación de Chile con el océano constituye un pasado, presente y futuro en el desarrollo del país. Por ello, lo que acontezca con los océanos en lo global es también de importancia para lo nacional. Ello, tanto por el aporte económico que ofrece el mar en pesca, comercio, conectividad con el mundo y actividad portuaria, como por su contribución ambiental en biodiversidad y cambio climático. Estos motivos son la razón por la cual Chile ha presentado un interés histórico por involu- 
crarse de forma activa en las iniciativas multilaterales que se han desplegado para establecer la sostenibilidad en el tratamiento hacia los océanos y que presentan un país consciente de la responsabilidad y vínculo que le compete en los océanos.

En tal contexto, las Áreas Marinas Protegidas (AMP) son un instrumento de uso como estrategia para la conservación y gestión de zonas marítimas al servicio de los Estados. El reconocimiento del aporte de éstas ya se ha consignado en tratados y protocolos, e incluso fue establecido en los Objetivos de Desarrollo Sostenible (ODS) de la Agenda 2030 de las Naciones Unidas (ONU), lo que muestra su valor universal.

A partir de ello, este escrito presenta como objetivo general proponer el concepto de "responsabilidad oceánica de Chile" y su aplicación en la gestión de las Áreas Marinas Protegidas como impulsor de una política exterior marítima que maximice los intereses nacionales desde su vinculación al desarrollo sostenible. Para ello, se expone la creación y desarrollo del concepto de "responsabilidad oceánica" como una propuesta innovadora, pero arraigada en una historia de contribuciones nacionales en la materia, y se busca aportar con un principio activo para hacer de Chile un referente oceánico, compatibilizando la conservación ambiental, los intereses marítimos y el desarrollo nacional.

El objetivo específico que se presenta es examinar la categorización de las AMP en Chile como Laboratorios Naturales, en un contexto multilateral que apunta a lograr tener océanos más sostenibles. Esto se realiza a partir de la vinculación de la acción del Ministerio de Relaciones Exteriores de Chile (MINREL) con los otros actores partícipes. Asimismo, en el plano metodológico, se aplica el enfoque inductivo, utilizando fuentes directas, como entrevistas a intelectuales y funcionarios públicos (estos últimos, citados anónimamente a petición de las partes, a fin de resguardar su identidad y de posibilitar que se expresen más abiertamente) y documentación oficial, además de recursos como investigaciones de organismos oficiales y bibliografía académica.

\section{La contribución de Chile al derecho del mar y el multilateralismo}

A partir de cómo se ha ido configurando el derecho del mar y las diversas iniciativas multilaterales es que la participación de Chile cobra preponderancia. En tal contexto, se debe enfatizar la vocación oceánica y capacidad propositiva de Chile que ha acompañado el proceso global desde su inicio. Allí entra la histórica Declaración Presidencial del 23 de junio de 1947 de Gabriel González Videla, en donde expresaba que "Chile confirma y proclama la soberanía nacional sobre los mares adyacentes a sus costas (...) para reservar, proteger, conservar y aprovechar los recursos y riquezas (...) declarándose desde luego dicha protección y control sobre todo el mar comprendido dentro del perímetro formado por la costa con una paralela matemática proyectada en el mar a doscientas millas marinas de distancia de las costas continentales" (González, 1947, p. 2).

Tal hito fue una declaración unilateral con repercusiones globales y un contenido que, además de soberanista, tenía un componente conservacionista, que fue luego acompañado de un movimiento regional, pues el 18 de agosto de 1952 la Declaración sobre la Zona Marítima es la activación de la Comisión Permanente del Pacífico Sur (CPPS) donde Chile, Ecua- 
dor y Perú (sumándose Colombia en 1979): “proclaman como norma de su política internacional marítima, la soberanía y jurisdicción exclusivas que a cada uno de ellos corresponde sobre el mar que baña las costas de sus respectivos países, hasta una distancia mínima de 200 millas marinas desde las referidas costas" (CPPS, 1952, p. 1). Esto constituyó un paso al reconocimiento internacional de una postura política sobre el mar que fue acogida en la Convención de Naciones Unidas sobre Derecho del Mar (CONVEMAR).

En particular, los acuerdos en el marco del sistema del Pacífico Sur han sido múltiples, dentro de lo que destaca el Protocolo para la Conservación y la Administración de las Áreas Marinas y Costeras Protegidas del Pacífico Sudeste, de 1989 (ratificado por Chile en 1993), que fue precursor en normar las AMP y sentó un precedente mundial en cuanto a proteger y preservar los ecosistemas marinos.

La Declaración Presidencial y la CPPS rindieron frutos en 1982 en la CONVEMAR, tal como detalla Fernando Zegers, diplomático chileno presente en las negociaciones: "Chile obtuvo en y mediante la CONFEMAR la, hasta entonces, lejana y casi inalcanzable consagración de la Zona Económica de 200 millas, la cual nuestro país fue el primero en declarar y promover" (Zegers, 2018, p. 540). De este modo, se derivó en que a nivel regional en 2009 se crease la Organización Regional de Ordenación Pesquera en el Pacífico Sur (OROP-PS). Su Convención, sobre la Conservación y Ordenamiento de los Recursos Pesqueros en Alta Mar en el Océano Pacífico Sur, está pensada para garantizar la conservación y uso sostenido de los recursos, a partir de los enfoques precautorios y ecosistémicos y el principio de compatibilidad.

De tal manera, el sistema del Pacífico Sur aseguró no sólo el principio precautorio que no figura en CONVEMAR, sino también buscó velar por principios de uso sostenible de los recursos pesqueros con un ordenamiento transparente y responsable, evitando la sobrepesca y promoviendo cooperación científica y transferencia tecnológica.

Este abanico de acuerdos y la participación de Chile en sus procesos y ratificaciones ${ }^{2}$, tras un periodo de normativización del ámbito jurídico, sufrió una reconfiguración en el siglo $X X I$, lo que llevó a un aumento de las actividades multilaterales promotoras de la acción sobre el enfoque jurídico. Ejemplo de esto es la Iniciativa Our Ocean II, realizada en Valparaíso en octubre de 2015, instancia en la cual los Gobiernos compartieron con organizaciones no gubernamentales (ONGs), académicos y la sociedad civil. En esta conferencia, cada parte expuso las acciones que se comprometía a cumplir, lo que sirvió a Chile para posicionarse en la diplomacia multilateral de los océanos.

\footnotetext{
2 Los tratados internacionales vinculados con la materia y suscritos por Chile son: CONVEMAR y su Anexo Parte XI; Convenio Internacional para la Seguridad de la Vida Humana en el Mar (SOLAS); Convenio Internacional para prevenir la contaminación por los Buques (MARPOL 73/78); Convención para la Conservación de los Recursos Vivos Marinos Antárticos; Acuerdo de Conservación por los Buques Pesqueros que Pescan en Alta Mar; Acuerdo del Estado Rector contra la Pesca llegal, No Declarada y No Reglamentada (INDNR); Convención de Conservación y Ordenamiento de los Recursos Pesqueros en Alta Mar en el Pacífico Sur; Acuerdo sobre Peces Transzonales y Altamente Migratorias; Código de Conducta de la Organización de las Naciones Unidas para la Alimentación y la Agricultura (FAO) para la Pesca Responsable; Plan de Acción FAO para Prevenir, Desalentar y Eliminar la Pesca INDNR.
} 
Aquella convergencia concretó el paso de Chile, desde una actitud política que fue (y es) activa en lo jurídico hacia una de acciones tangibles. Esto lo reflejó en el anuncio de dos AMP de una vasta extensión en sus islas oceánicas, con su ratificación al Acuerdo de Peces Transzonales y Altamente Migratorios, entre otros hechos, que le posicionaron en buena plataforma para su visibilización internacional. Ello se reforzó en la Declaración Because The Ocean del 29 de noviembre de 2015. Allí, diferentes gobiernos enfatizaron los beneficios que significan los océanos, para sentar tres pilares: 1 ) apoyar la investigación sobre los nexos entre océanos y cambio climático, 2) reafirmar el ODS 14 y su implementación, y 3) promover un Plan de Acción para los Océanos. Declaración, de la cual Chile es uno de sus 24 firmantes, lo que expresa su compromiso con el multilateralismo.

Esta nueva modalidad de acción mezcla normativa jurídica, multilateralismo y la participación en instancias mixtas (con Estados y otros actores), y refleja la realidad que vivencian los océanos, en la medida que, además de los países, otros actores tienen influencia y preocupaciones. Por esta razón no fue extraño que, en septiembre de 2017, Viña del Mar fuese sede del IV Congreso Internacional de Áreas Marinas Protegidas (IMPAC IV), lo que indica que, con el fomento a la conservación ecosistémica marina en las AMP, se busca fortalecer los esfuerzos globales y las alianzas transversales en aras del tema oceánico, reafirmando la intención de cumplir la Meta 11 Aichi del Plan Estratégico para la Diversidad Biológica 2011-2020 del Convenio sobre la Diversidad Biológica (CDB) y las metas del ODS 14.

La evidencia aquí presentada denota que Chile ha tenido una línea de continuidad en su preocupación por el tema oceánico y que es producto de una reflexión consciente. En un primer momento, apuntó hacia una afirmación de soberanía para derivar wn una visión donde primó la explotación de recursos, pero que, a medida del avance del conocimiento científico, fue derivando a la senda actual de compatibilidad entre uso y protección del ambiente marino.

\section{La Política Oceánica Nacional (PON)}

Una de las consignas de la Política Exterior Marítima (PEM) es que Chile es un país con vocación oceánica, sostenida por la historicidad, desde la visión de Bernardo O’Higgins sobre el valor del dominio del mar e impulsor del poderío naval, pasando por el combate naval de lquique, hasta los actos soberanos de colonización en zonas extremas, entre otros. Junto a ello, mientras el Chile terrestre cubre $756.096 \mathrm{~km}^{2}$, el Chile marítimo asciende a 3.403 .235 $\mathrm{km}^{2}$, que se desglosan en $120.827 \mathrm{~km}^{2}$ de mar territorial, $131.669 \mathrm{~km}^{2}$ de zona contigua y $3.159 .739 \mathrm{~km}^{2}$ de la zona económica exclusiva (ZEE). Tal dimensión marítima refuerza la lógica de pensar a Chile como un país oceánico, prestando atención al desarrollo de una PEM, pues los océanos son espacios de frontera y uso internacional. Esta es la motivación de Chile para crear una Política Oceánica Nacional.

Pero la creación de la PON no es algo nuevo. El general Ramón Cañas a mediados de siglo ya planteaba la urgencia de una política oceánica centrada en el Pacífico, propiciando un ensanchamiento del horizonte geográfico nacional, puesto que tal era el destino y misión de Chile (Cañas, 1954, p. 148). Esa era su apreciación para el venidero cambio de época, en 
el cual (del Atlántico norte al Pacífico sur), Chile tenía las condiciones de convertirse en una futura potencia del Pacífico Sur-Antártico.

Asimismo, a fines del siglo pasado, el almirante Jorge Martínez manifestaba "la necesidad de una doctrina oceanopolítica fundada en la influencia y gravitación permanente que ejercen los espacios oceánicos en la formulación de las políticas nacionales" (Martínez, 1994, p. 1), con el fin de integrar el territorio oceánico nacional, a partir de unos objetivos relacionados con la realidad marítima de Chile, con políticas metodológicas y sistemáticas aplicadas. No obstante, tales impulsos no fueron concretados y fueron sólo enfocados desde la defensa nacional, por lo que hacía falta un diálogo con otras instituciones del Estado.

Recién en 2014 la idea empieza a cobrar nuevos bríos, esta vez, liderada por MINREL, la que da forma al Consejo de Ministros para el desarrollo de Política Oceánica (CMDPO) formado por las carteras de Economía, Defensa, Relaciones Exteriores y Medio Ambiente, con una visión articuladora institucional. Se apuntaba a consolidar una política de Estado para los temas marítimos y ser el noveno país en contar con Política Oceánica Nacional ${ }^{3}$. De tal forma, los esfuerzos se enfocaron en crear un insumo que identificara leyes nacionales y convenios internacionales que rigen el actuar marítimo de Chile, y construir ejes estratégicos, orientaciones, prioridades e intereses en el océano.

En dicho documento se incluyó la necesidad de fortalecer un modelo institucional de gobernanza para los ámbitos de trabajo en materia oceánica. Ello, con el fin de optimizar la coordinación, información y las decisiones para ser capaces de articular los intereses sectoriales representativa y equilibradamente de modo de lograr los objetivos discutidos. Junto a eso, se reconoce el valor de promover la participación público-privada. Así se aspira a evitar la dispersión institucional y las duplicidades sobre el quehacer en el océano, apuntando hacia una gobernanza que fomente el encadenamiento intersectorial.

En dicha línea, los países con PON han dado distintas respuestas a la necesidad de tal intersectorialidad. Un ejemplo es Canadá, una de las pocas naciones con un ministerio para temas oceánicos (Ministry of Fisheries and Oceans), mientras países como EE.UU. y Colombia han optado por una comisión nacional coordinadora, pero sin pasar a llevar la competencia de cada institución con funciones específicas en el mar. En tanto, en Chile la misión del proceso de convergencia quedó en manos del MINREL. Pero la PON no explicitó a quien compete la conducción a futuro. Recién en 2020, a partir del documento "Mares de Chile, Visión 2040", publicado por el MINREL, se reconoce la valía del Consejo para implementar, actualizar, dar seguimiento y cumplimiento de las acciones que el país estableció en la PON, por lo que se propuso darle carácter permanente a su trabajo para evitar el riesgo de que se diluya entre otras prioridades de cada ministerio. Sin embargo, aún queda pendiente que ello sea operativizado.

Ahora bien, respecto de la situación de los actores envueltos en temas oceánicos, la PON indica que hay participación de los cuatro ministerios que componen el Consejo, junto con la Armada, el sector académico y científico desde el Consejo Nacional de Investigación, 
Ciencia y Tecnología (CONICYT) ${ }^{4}$, y consultivamente de los privados, ONGs y la sociedad civil. Pero haciendo un análisis sobre los actores institucionales y sus competencias, podría incluso plantearse el involucramiento indirecto de otros organismos en el fomento de la PON y su aporte al desarrollo nacional. Por ejemplo, el CONICYT tiene fondos reservados para la investigación oceánica en temas como recursos genéticos marinos, el Ministerio del Deporte ha generado un plan de fortalecimiento en deportes acuáticos y el Ministerio de Minería, para los temas de explotación minera en los fondos marinos nacionales o alta mar.

El documento PON explicita nueve objetivos nacionales entre los que destacan para esta investigación, el $n^{\circ}$. 1: "la conservación del océano y su biodiversidad, implementada bajo un marco de equilibrio intersectorial que armonice el desarrollo social, el crecimiento económico y la gestión del medio ambiente marino y los recursos que contiene, para beneficio de la sociedad actual sin poner en riesgo el desarrollo de las futuras generaciones" (CMDPO, 2018, p. 15); el n. 5: "Acorde con el cumplimiento del Derecho Internacional del Mar y los Objetivos de Desarrollo Sostenible de las Naciones Unidas, posicionar a Chile como actor relevante en los asuntos oceánicos internacionales" (CMDPO, 2018, p. 15); y el séptimo: "Promover la conciencia y la comprensión pública sobre el océano, fortaleciendo los vínculos que poseen los ciudadanos con el mismo" (CMDPO, 2018, p. 15). Se posicionan así como áreas sectoriales a: 1) la conservación del océano y de sus recursos, 2) desarrollo económico, 3) seguridad y océano;,4) océano y territorio, y 5) el desarrollo científico.

No obstante, un ítem que la PON omite en su composición es su vigencia, a diferencia de otras, como la Política Energética de Chile 2050. La PON no hace referencia a cuándo debe ser revisada o actualizada, dejando en vilo su contextualización a las futuras necesidades. De cualquier modo, debe reconocerse que la PON integra al océano de manera multidimensional a la estrategia nacional de desarrollo, pensando bidireccionalmente: "las aproximaciones top-down y bottom-up deben complementarse mutuamente, como una estrategia para medir las oportunidades políticas y mejorar los compromisos sociales en las iniciativas de conservación. Debe haber un reconocimiento de la interacción entre AMP y el amplio sistema socio-ecológico, y la incorporación de la percepción de los stakeholders en la gobernanza de las AMP" (Araos \& Ther, 2017, p. 70).

En lo medular, el documento es una fuente moderna sobre las relaciones de un Estado para con el océano, que presenta fortalezas como la valoración de la necesidad de la educación como foco de concientización oceánica. También se enfatiza la innovación como el factor clave para una economía diversificada y sostenible, sumado a que es prioridad, porque la ciencia y tecnología aplicadas son clave para una capacidad de producción con mayor valor agregado. Como se explica: "el desarrollo de capacidades de innovación en las comunidades requiere de la contribución de diversos actores del sistema nacional de innovación, en particular de la academia" (CMDPO, 2018, p. 141).

Al mismo tiempo, posee puntos cuyo enfoque podría ser profundizado. Por ejemplo, no figuran las cuatro líneas de trabajo de los comités de preparación para la negociación del

4 En 2020, CONICYT pasó a denominarse Agencia Nacional de Investigación y Desarrollo de Chile (ANID), dependiente del Ministerio de Ciencia, Tecnología, Conocimiento e Innovación, creado en 2018. 
nuevo tema que el derecho del mar aspira a normar: el Biodiversidad Más Allá de la Jurisdicción Nacional (BBNJ, por sus siglas en inglés). No se tratan la prospección marina y recursos genéticos marinos, las regulaciones para estudios de impacto ambiental, ni la transferencia de capacidades y tecnologías. Además, el tema de las AMP en alta mar no está desarrollado.

Por otra parte, se haría necesario un desarrollo mayor de la metodología, pues, aunque se identifican falencias y mejoramientos, mucho de lo que figura en la PON como acciones, realmente son objetivos, al no explicitar cómo llevarlos a cabo -sobre cómo fortalecer la institucionalidad en temas del mar, ya que no se incluye cómo se realizaría ni bajo qué formato. Tampoco se ahonda en las AMP, que sólo se mencionan dos veces en todo el escrito, pero sin hacer la bajada a implementación de estas tras su declaración como áreas protegidas. De hecho, no se toca la cuestión del AMP Antártica en el Dominio 1 que Chile y Argentina están impulsando, que no se regiría por ley nacional sino por la Convención sobre la Conservación de los Recursos Vivos Marinos Antárticos. Eso hace parecer que en el documento prima un discurso generalista por sobre uno más inspirado en los desafíos que Chile tiene.

Finalmente, otra área que fue poco desarrollada es la diversidad entre regiones. Hace falta ver la particularidad de cada región, pues poseen ecosistemas, problemas y necesidades distintas. Podrían realizarse apartados por macrozona oceanográfica o impulsar anexos de políticas oceánicas regionales, ya que una mirada generalista, si bien es importante para coordinar, puede pecar de poco funcional para cada uno de los sectores, lo que podría terminar convirtiendo a la PON en más declarativa que práctica.

\section{Desarrollo del concepto de responsabilidad oceánica de Chile}

Como se indicó en apartados anteriores, Chile ha poseído una preocupación histórica por los temas oceánicos, que ha estado presente en su diplomacia y acciones multilaterales, por lo que el concepto de "responsabilidad oceánica" aquí propuesto deriva de esta vocación oceánica que el país manifiesta sostenidamente, a la vez que aporta una visión innovadora al recoger dinámicas de una gestión pública moderna, más inclusiva e intersectorial, que busca propiciar la articulación de una política de Estado a largo plazo. Es considerando que la PON carece de principios que guíen los objetivos que expone, que se pone a debate este concepto; para que los esfuerzos del país no sean dependientes de la voluntad de las autoridades de turno, sino se mantengan como prioridad y contribuyan a guiar el quehacer nacional.

Pero hay que hacer la aclaración: la responsabilidad oceánica no debe constituir una obligatoriedad jurídica para el Estado en lo internacional; tiene un enfoque político que se aprecia por su valor ético. Por ello, no se contrapone al pleno resguardo de la soberanía, las obligaciones bilaterales o internacionales que haya adquirido el país y que componen la base mundial de la gobernanza oceánica (siendo CONVEMAR el eje). Más bien, busca sumar las áreas que no están reguladas, impulsando a que sean normadas, como lo que acontece con el BBNJ, pero utilizando también el soft law como instrumento que propicie una base de países con posturas similares a la que luego puedan sumarse otros, con la aspiración de 
crear normas o costumbres. La participación chilena en instancias como Our Ocean o fal irmar el Because the Ocean sería una forma de reafirmar tal responsabilidad.

La propuesta de lo que la responsabilidad oceánica debe significar para Chile es la siguiente: la acción y promoción continua de intereses de política exterior marítima vinculados a la gestión sostenible de los recursos y ecosistemas oceánicos, a través de la diplomacia y políticas públicas. Es un principio, en razón, de ser una idea fundamental que sirve como inspiración y orientación central de una acción valiosa de ser realizada. Es una responsabilidad, pues "tiene relación no con responder-a (una pregunta), sino responder-por (una persona)" (Dussel, 2011, p. 106), en este caso, responder-por los intereses y bienestar del país, las personas y el planeta. Es oceánico, porque apunta a la acción humana en aras de océanos sostenibles, limpios y resilientes, frenando los efectos de la contaminación, el cambio climático, la sobreexplotación y la pesca INDNR.

Esta cuestión debe ser abordada bajo la lógica de "Principio Activo", tal y como la Embajadora de Chile ante los Países Bajos, María Teresa Infante, indica sobre estas responsabilidades (Infante, M., comunicación personal, 12 de enero de 2018), para que apunte al desarrollo, fortalecimiento y potenciamiento de las capacidades estatales en la materia. Esto posee una similitud con la connotación que se le da en los "Principios de la Política Exterior Chilena" a la "Responsabilidad de Cooperar", donde Chile asume el compromiso político de cooperar, a través de sus recursos técnicos y humanos pero sin obligatoriedad jurídica.

Este principio debe vislumbrar la dimensión interna e internacional en interacción bidireccional. En lo internacional, respecto del Estado como sujeto con derechos soberanos sobre su espacio marítimo, con la participación multilateral y cooperación que implica con los otros actores del escenario global. En lo doméstico, desde una desagregación que visibilice los organismos e instituciones a las que conciernen los temas oceánicos, apuntando hacia una intersectorialidad que coordine actores más allá del Estado, que incluya la sociedad civil y el sector privado (Espaliat, A., comunicación personal, 9 de abril de 2018). Ello configura a la responsabilidad oceánica como un concepto bidireccional, en el sentido que la dimensión externa se nutre de la gestión de políticas públicas domésticas y la política interna se fortalece a partir de una diplomacia capaz de hacer convergencia con las tendencias globales que ponen énfasis en la coordinación y cooperación internacional en el cuidado de los recursos y ecosistemas oceánicos.

Sin ser la responsabilidad oceánica meramente una noción ambientalista que aspira a proteger los océanos, pues debe ser interpretada como un concepto multidimensional y con fuerte arraigo a los intereses marítimos nacionales; deba servir como concepto catalizador de Chile como referente oceánico. Debe ser funcional a que el país se convierta en un actor protagónico de la gobernanza oceánica global, con el objetivo de hacer permanente la proyección de Chile hacia las altas esferas de la toma de decisiones de la política multilateral. Pero, sobre todo, debe materializarlo en acciones, que, en el ámbito de la política exterior, apunten a posicionarlo en la agenda, comunicarlo a los otros países e incorporarlo a la diplomacia como un concepto que haga del quehacer internacional de Chile, un aporte al desarrollo. 
Para construir su imagen y convertirse en referente en temas oceánicos, Chile debe potenciar la capacidad de influir a través de su acción como un ejemplo, un rol propositivo -tal como sucedió con la creación de la ZEE- y articulador de iniciativas tendientes al uso sostenible de los océanos y el desarrollo del país, la región y el mundo. Para tales fines, el posicionamiento internacional como país debe venir desde la capacidad de conducción de la política oceánica integrada en función del ejercicio de tal responsabilidad, obtener beneficio político de ello y catalizarlo estratégicamente hacia la configuración de Chile como actor clave en el área.

De hecho, la noción de referente para pensar a Chile no es algo tan lejano en los temas oceánicos, pues: "se encuentra entre las cinco primeras potencias pesqueras del mundo en cuanto a captura de peces y en octavo lugar en producción de acuicultura y está calificado en el décimo puesto de índice de gobernanza costera" (Aimone, 2016, p. 14), a lo que habría que sumar que, en 2019, Chile fue el cuarto mayor usuario del Canal de Panamá ${ }^{5}$, tras EE.UU., China y Japón. Esto denota que el país posee relevancia global en materia de economía, comercio y recursos marinos.

De allí que, tal como señalan desde la Dirección de Medio Ambiente del MINREL (identidad resguardada, comunicación personal, 20 de febrero de 2018), el desafío es grande cuando implica el compatibilizar ingresos y recursos con la responsabilidad en temas como uso sostenible y conservación oceánica, ya que sencillo sería para un país sin mayor capacidad pesquera convertirse en un referente de protección ecosistémica. Por tanto, siendo Chile potencia en tal área, su legitimidad se fortalece si logra posicionar armónicamente lo ambiental con lo económico y superar "la dicotomía entre explotación y conservación, y los vaivenes que sufrirá nuestra política entre uno y otro carril a través de los años, situación que parece finalmente dilucidarse (...) que va a colocar a Chile como uno de los países pioneros, una vez más" (Ortega, 2015, p. 178).

Es más tangible ver el lugar de Chile en exportación de recursos pesqueros o recaladas en sus puertos, que dilucidar su posición en materia ambiental. Para tales fines, resulta útil el Ocean Health Index, la clasificación internacional sobre la salud de los mares (metodología que utilizan Colombia, Ecuador y Venezuela para sus evaluaciones), que ubicó a Chile en el puesto 54 en $2018^{6}$, en un análisis que toma un amplio abanico de temas ${ }^{7}$. Pero es un puesto lejano de países de vanguardia. Chile es fuerte en economía costera (mayormente por la actividad portuaria), biodiversidad y limpieza de océanos, pero se encuentra débil en temas de provisión de alimentos, turismo y recreación, y peligrosamente débil en productos naturales, sin siquiera ser categorizable en protección costera y contaminación carbónica.

5 Disponible en: https://micanaldepanama.com/wp-content/uploads/2019/10/10-PrincipalesPaises.pdf

6 Véase: http://www.oceanhealthindex.org/region-scores/scores/chile-+-easter-island

7 Categorías y puntuación de Chile (sobre un máximo de 100 puntos): 1) Provisión de alimentos 63; 2) Oportunidades para pescadores artesanales 89 ; 3) productos naturales 18 ; 4) Almacenamiento de carbono s/i; 5) Protección costera s/i; 6) Economía y medios de vida costera 100; 7) Turismo y recreación 32; 8) Sentido de pertenencia 80; 9) Aguas limpias 94; 10) Biodiversidad 95. 
Esto reafirma la necesidad de proteger la biodiversidad y tener mares limpios, que se tornan una fortaleza única a nivel mundial, y expone la urgencia de desarrollar mecanismos que mejoren la amenaza de la sobreexplotación pesquera y fomenten el valor agregado a la acción de las comunidades. Identificadas tanto fortalezas como debilidades, el país podría establecer como metas, para 2020, mejorar sus áreas para alcanzar el puesto 50 y para 2030, estar entre las 20 naciones con océanos más sanos. Lo anterior, no solo por ascender en un ranking, sino porque el ecosistema lo necesita, porque ayuda al desarrollo y porque da legitimidad a Chile para posicionarse globalmente.

Ahora bien, la responsabilidad oceánica, para ser una contribución para la PON, debe tener clarificados los tópicos transversales que debe desarrollar para una fuerte línea de continuidad. Es posible identificar cinco áreas relacionadas que hacen de la responsabilidad oceánica un concepto coherente con la gobernanza oceánica, vinculado a sectores fuera de lo estatal: la ciencia y tecnología; la sociedad y comunidades; y desde lo estatal, la triada: regulación legal, implementación de políticas públicas y rendición de cuentas.

La propuesta aquí desarrollada, desde la visión de conservación y uso sostenible, es a su vez multidimensional, al aspirar a tocar las distintas amenazas que enfrentan los océanos. La Responsabilidad Oceánica del país requiere detener la acción contaminante (marina y de origen terrestre) del ser humano sobre los océanos, pues no pueden tenerse océanos sanos si estos no están limpios. Esto involucra acciones preventivas y hacerlos más resilientes. También infunde a fortalecer los océanos como agentes contra el cambio climático, es decir, dar paso a iniciativas como la reforestación de los bosques marinos. E involucra otros tópicos, como detener la escala e intensidad de la sobreexplotación de recursos que se lleva a cabo, o enfrentar la pesca INDNR con todas las herramientas disponibles.

Por esto, se propone la configuración de un nuevo ítem de "Intereses de la Política Exterior de Chile", que podría llamarse "Promover la Responsabilidad Oceánica e Intereses Marítimos Nacionales", porque en el actual ítem figura "Promover los intereses marítimos y antárticos de Chile". Esos temas, aunque ligados, no se entrelazan en todas sus acciones ni se pueden pensar iguales. Así, resultaría conveniente una separación para dar énfasis, por separado, a cada arista. Además, suma a los intereses marítimos, la visión de responsabilidad oceánica, el concepto que este escrito propone y pone en debate para generar un principio que dé continuidad a la acción y fortalezca la base de pensamiento de la PON, sosteniéndola sobre los pilares de la protección y uso sostenible de los océanos, al servicio del interés nacional.

Estas áreas aspiran a armonizar la responsabilidad oceánica como concepto integral que apunte al desarrollo sostenible. Este concepto debe ser contextualizado a la realidad chilena para poder servir a sus intereses. Esto hace que, en la actualidad, utilizarlo deba estar vinculado a las temáticas que el país estableció como prioritarias en los últimos años, a saber: Áreas Marinas Protegidas. Este eje ha sido el puntal de la gestión que lidera el Estado, guiado por el MINREL. Las razones de esta decisión son porque las AMP son "un catalizador de las funciones que cumple el océano" (Laborda, 2018, p. 616).

Así, la responsabilidad oceánica puede establecerse como el argumento que dé el sentido al magno esfuerzo conservacionista de las AMP en el país, pero desde la nueva óptica 
que entregue una gestión activa y no sólo nominal. Allí se hace funcional que la PON articule nacional e internacionalmente la captación de capacidades, conocimientos y habilidades vinculadas a la ciencia y tecnología en temas oceánicos, haciéndolo una piedra angular que permita reforzar a Chile como un Laboratorio Natural. Esta idea implica una nueva relación holística y ser activos participantes en los desafíos científico-tecnológicos globales, pero a su vez, está firmemente arraigada en las necesidades de las comunidades y la sociedad.

\section{La aplicación de la responsabilidad oceánica en la gestión de las AMP}

Sobre las Áreas Marinas Protegidas, si bien no existe una acepción única y consensuada, la Unión Internacional para la Conservación de la Naturaleza entiende por ellas: "un espacio geográfico claramente definido, reconocido, dedicado y gestionado a través de medios legales u otros igualmente efectivos, en aras de lograr la conservación a largo plazo de la naturaleza y los servicios ecosistémicos y valores culturales asociados a ésta" (OCDE, 2017, p. 31). Pero no existe una normativa global que les defina y/o clasifique, siendo labor de cada país cómo aplicar su jurisdicción nacional para sus AMP.

En cuanto a convenios internacionales, el Convenio sobre la Diversidad Biológica de 1992 en su artículo 8 establece como una forma de conservación in situ a las áreas protegidas (incluidas las terrestres y marinas), que serían lugares donde se toman medidas especiales para conservar la biodiversidad, con directrices tendientes a reglamentar y administrar las zonas, para garantizar su conservación y uso sostenible. Asimismo, en 2010, las Metas de Aichi propusieron, en su Meta 11, que para 2020 hubiese en el mundo 10\% de AMP, lo que, dado que no se cumplió, se recoge en la meta 5 del ODS 14 de la Agenda 2030.

En el contexto del sistema regional de la CPPS, anterior a lo internacional, en 1989 se estableció el Protocolo para la Conservación y Administración de las Áreas Marinas y Costeras Protegidas del Pacífico Sudeste. Allí, en su artículo 2, los Estados, entre ellos Chile, se comprometieron a "establecer bajo su protección, en la forma de parques, reservas, santuarios de fauna y flora u otras categorías de áreas protegidas. En estas áreas se establecerá un manejo íntegro, sobre la base de estudios e inventarios de sus recursos, con miras al desarrollo sostenido de ellos, prohibiendo toda actividad que pueda causar efectos adversos sobre el ecosistema, fauna y flora, así como su hábitat" (CPPS, 1989).

Al alero de esta base jurídica internacional, Chile confirma que su labor por las AMP no es algo surgido del último tiempo, pues a la firma del Protocolo de 1989 se le suman otros hitos, como la declaración de Islas Salas y Gómez como santuario de la naturaleza en 1976, la incorporación de los parques y reservas marinas a la ley 18.892 de Pesca y Acuicultura de 1991, y la ley 20.657 de 2013 que incorpora los enfoques precautorio y ecosistémico.

Así, su estructura normativa se basa sobre el reconocimiento de cinco tipos de AMP: 1) parque marino, 2) reserva marina, 3) área marina costera protegida de múltiples usos (AMCP-MU), 4) espacio costero marino de pueblos originarios (ECMPO) y 5) santuario de la naturaleza. La ley 18.892 crea el reglamento sobre parques y reservas marinas, donde se señala que el primero es "área específica y delimitada destinada a preservar unidades ecológicas de interés para la ciencia y cautelar áreas que aseguren la mantención y diversidad de especies hidrobiológicas, como también aquellas asociadas a su hábitat [y el segundo es] 
(...) un área de resguardo de los recursos hidrobiológicos, con el objeto de proteger zonas de reproducción, caladeros de pesca y áreas de repoblamiento por manejo" (Decreto 238, Ministerio de Economía, Fomento y Turismo, p. 2). Al provenir del Ministerio de Economía, queda bajo la responsabilidad del Servicio Nacional de Pesca (SERNAPESCA).

En tanto, la tercera categoría es el AMCP-MU, que es una figura ambigua a cargo del Ministerio de Medio Ambiente (MMA), a partir de sus facultades en la ley 19.300, artículo 70 , letra $C$, de proponer las políticas, planes, normas y fiscalizar las áreas marinas costeras protegidas de múltiples usos. No obstante, las AMCP-MU no poseen ni reglamento, ni definición conceptual, abriendo paso a interpretaciones y, aunque se les denominan áreas costeras y hasta los 80 metros desde la línea de base es jurisdicción de la Armada, las AMCPMU pueden ser más extensas, como en la Isla de Pascua, donde llega a la milla 200.

Mientras "parques y reservas marinas" son restrictivos en sus usos, las AMCP-MU, al no tener reglamento, dejan abierto el uso posible. La claridad la dio un pronunciamiento de la Contraloría en 2013 que indica que ex ante, no se puede prohibir ninguna actividad, siendo la única regla que éstas sean compatibles con los fines de la conservación ambiental. Esto abre la opción a actividades como la acuicultura para funcionar dentro de las AMCP$\mathrm{MU}$, con toda la polémica de ello, por la implicada contaminación de aguas por vertimiento de efluentes, pesticidas y medicamentos (OCDE \& CEPAL, 2016, p. 50).

Además, deben mencionarse los ECMPO, creados para las comunidades indígenas de identidad marino-costera, en iniciativa de la Corporación Nacional de Desarrollo Indígena (CONADI) en la ley 20.249, que hace que la Subsecretaría de FF.AA. le otorgue los espacios a la Subsecretaría de Pesca, quien suscribe un convenio de uso con la comunidad. Esto se suma al santuario de la naturaleza, desde la ley 17.288 de 1970, extensible a áreas terrestres o marinas.

Actualmente Chile posee $42 \%$ de su ZEE con alguna figura de $\mathrm{AMP}^{8}$, lo que pone al país a la vanguardia a nivel mundial en porcentaje de áreas protegidas. Este proceso fue vertiginoso, pues en 2004 se creó el primer parque marino, "Francisco Coloane"; para 2013, 4\% del territorio marítimo chileno estaba protegido y para 2016 , ya era $12 \%$. Esto refleja cómo los mares pasaron a ser la prioridad (una forma de responsabilidad oceánica), con un enorme valor para el país y el mundo.

Estos datos han visibilizado a Chile en materia de AMP, pero cabe mencionar que la declaración de AMP no es el paso final sino la etapa inicial del proceso. Para que el país sea considerado referente, el compromiso político debe extenderse a su implementación. Ese es el próximo desafío, pues de las trece AMCP-MU, tres poseen plan de administración; de los diez parques marinos, sólo uno posee; de las cinco reservas marinas, cuatro cuentan con este plan; y de las nueve EMCPO, ocho tienen su plan de gestión, y dos de los diez santuarios de la naturaleza en área marina. Estos planes son importantes para dar cabal cumplimiento a la Meta de Aichi 11, que indica que $10 \%$ de áreas protegidas deben ser administradas de manera eficaz y equitativa.

8 Para revisar la composición, tamaño y lugar de las AMP: http://areasprotegidas.mma.gob.cl/ 
Junto a ello, la mayor parte de dichas áreas son en las islas oceánicas. Esto es positivo para el resguardo de estas ecorregiones de alta biodiversidad, pero, por otra parte, como se observa desde el MMA (identidad resguardada, comunicación personal, 13 de febrero de 2018), hacen falta AMP de mayor representatividad de los ecosistemas nacionales, como los costeros, más vulnerables a la acción antrópica. Otra arista compleja es la designación de zonas como AMP. Desde SERNAPESCA (identidad resguardada, comunicación personal, 2 de marzo de 2018) indican que, aunque se piden planes de administración una vez creada el AMP, no se realizan estudios científicos previos sobre por qué tal zona debe ser declarada AMP. Eso puede hacer que la decisión se aleje de lo técnico para acercarse a lo político.

En la Dirección General de Territorio Marítimo y Marina Mercante (DIRECTEMAR) (identidad resguardada, comunicación personal, 22 de marzo de 2018) consideran relevante que lo político se vincule a lo técnico, fortaleciendo el Comité Nacional de Áreas Protegidas para evitar que se puedan declarar zonas sin previas bases técnicas que les avalen. Prefieren que se opte por no declarar zonas si no está la certeza de que existen las capacidades humanas y logísticas para implementarlas, o si no se crea con el consenso de las comunidades locales.

Esto es de relevancia, pues la OCDE en su estudio sobre AMP indica que existen tres categorías de costos asociados a las AMP 1) costos directos, que refiere al costo de establecer y operativizar el AMP, lo que va desde la planificación de actividades hasta la aplicación y control del área, además de la contratación de personal; 2) costos indirectos, alusivo a las consecuencias paralelas al AMP y que pueden ir desde desplazamientos más largos para las flotas pesqueras hasta grupos sociales disgustados creándose conflictos socioambientales; y 3) la siguiente mejor alternativa que pudo haber habido, y que ya no es realizable por existir el AMP, es ver otros usos posibles para la zona que pudieron haber sido rentables, como los ingresos por pesca o explotación de hidrocarburos (OCDE, 2017, p. 53) y aunque ello es difícil de estimar a priori, debe tenerse en consideración. La OCDE postula dos herramientas para la toma de decisiones sobre AMP: 1) estudio de valuación, utilizado para estimar los beneficios de declarar un AMP, y 2) el análisis costo-beneficio (OCDE, 2017, p. 62).

Tanto las tres categorías de costos como las dos herramientas para las políticas públicas son útiles para Chile. Resulta importante que cada plan de gestión de una nueva área incluya los costos asociados a su implementación, ya que existe la siguiente gran problemática: "muchas áreas protegidas carecen de suficientes recursos financieros y humanos, y sus planes de gestión solo se ejecutan en forma parcial, o están incompletos o desactualizados. Pese a los esfuerzos, es probable que Chile no cuente con planes de administración y gestión operacionales en todas las áreas protegidas hasta 2050" (OCDE \& CEPAL, 2016, p. 20). Una responsabilidad oceánica implica contar con los planes y recursos necesarios para el pleno desarrollo de cada AMP, lo que deriva hacia el tema presupuestario, y que en Chile poseen escasos fondos, con apenas 2 dólares por hectárea (comparado con los 16 por hectárea que destina Costa Rica):

“En 2012, la totalidad de los recursos financieros disponibles para las áreas protegidas oficiales rondaba los 41 millones de dólares (...) La CONAF [Corporación Nacional Forestal] recibe el grueso de los fondos asignados a las áreas protegidas, 72\%, (...) para administrar el SNASPE [Sistema Nacional 
de Áreas Silvestres Protegidas del Estado]. En cambio, el MMA recibe un $3 \%$ para administrar los santuarios de la Naturaleza, mientras que el SERNAPESCA recibe un ínfimo $0,3 \%$ para administrar las áreas protegidas marinas" (OCDE \& CEPAL, 2016, p. 254).

Esta situación, acorde con la nueva realidad del 42\% de ZEE con AMP, debiese cambiar, ya que, como señalan desde SERNAPESCA (identidad resguardada, comunicación personal, 2 de marzo de 2018), el aumento de AMP a nivel nacional no significó un incremento del presupuesto del Servicio para su implementación. Esto se suma a que velar por las AMP no es su misión originaria. Ello se Complejiza además, porque la ley 18.892, en su artículo 116 sobre infracciones por pesca INDNR, no diferencia sanciones entre infraccionar en cualquier zona o en un AMP.

La implementación y vigilancia de las AMP es algo que la OCDE y desde el MMA (identidad resguardada, comunicación personal, 13 de febrero de 2018) recomiendan se realice desde el Servicio de Diversidad Biológica y Áreas Protegidas (SBAP), aprobado en 2019 (pendiente en su forma de implementación) como el organismo responsable de una función que CONAF o SERNAPESCA realizan de manera liminar. No obstante, SERNAPESCA considera que el SBAP no necesariamente vendría a solucionar estas cuestiones, puesto que no contaría con presupuesto ni presencia regional y/o local, siendo su misión delegar, y seguiría siendo el Servicio el encargado de fiscalizar las AMP. Pero, de todas formas, sería un gran aporte para la coordinación integral y una ayuda en la confección de planes de administración en menor tiempo, y se posicionaría como una herramienta modernizadora del conservacionismo.

Aún con todas las falencias que puedan vislumbrarse, el sistema de AMP de Chile es el quinto del mundo y cumple con creces las metas que la comunidad internacional puso. "Las AMP cobran un rol fundamental y determinante en el combate efectivo del cambio climático y además contribuyen en la preservación de la biodiversidad marina, donde destacan los recursos pesqueros que han sido por años objeto de una fuerte presión de pesca en los océanos" (Laborda, 2018, p. 616). Así, la responsabilidad oceánica se entrelaza con las AMP, pero no sólo en la declaración de estas zonas, sino que parte de tal responsabilidad está en implementarlas debidamente y planificar los gastos concretos que significa ponerlas en marcha, para evitar que se conviertan en "Áreas Marinas Protegidas de papel”.

Ahora bien, existe cierta cautela desde algunos sectores, respecto de las AMP, por la consideración de que pueden afectar la economía nacional, cercando las pesquerías, frenando la explotación de recursos marinos como fuente de ingresos para el país. Frente a tal visión, hay formas de lograr lo que Ortega (2015) llama un equilibrio de conservación y explotación. En primer lugar, científicamente está comprobado el efecto rebalse que poseen las AMP, que permite incrementar los bancos de recursos en las zonas no protegidas, aumentando el tamaño de las especies y acelerando su reproducción (OCDE, 2017, p. 42).

En segundo lugar, si bien es cierto que el $42 \%$ de la ZEE está bajo alguna figura de AMP, sólo $0,3 \%$ corresponden a la ZEE del Chile continental, que es donde se realizan las actividades de 25 de las 26 pesquerías de Chile (la excepción es la pesquería de langosta de Juan Fernández e islas oceánicas), por lo que, en estricto rigor, el país no está cercenando áreas 
propias de la pesca. De hecho, la escala e intensidad de la explotación pesquera en Chile actualmente es crítico. De las 26 pesquerías: " 1 pesquería califica en estado de Subexplotación, 8 califican en Estado de Plena Explotación, 7 califican en Estado de Sobrexplotación, 9 se consideraron Agotadas o colapsadas y 1 es indeterminado debido a la condición del recurso" (SUBPESCA, 2018, p. 90). Justamente, la única en subexplotación es la de la langosta de Juan Fernández e islas oceánicas, donde los pescadores han generado un proceso bottom-up de conservación y uso sostenible, que actualmente generó un AMP.

Complementariamente, en caso de que genere suspicacia que la protección de Chile permita que justo en el límite exterior los pesqueros se aprovechen de la conservación de las AMP, para explotar las especies migratorias, existen 2 alternativas. Por una parte, el Acuerdo de 1994 relativo a la conservación de poblaciones de peces transzonales y migratorios apunta a ordenar su explotación. Por otra parte, el Acuerdo de 1989 de la CPPS, en su art. VI explicita que se establecerán, alrededor de las áreas protegidas, zonas de amortiguación, en las cuales los usos puedan ser regulados con el fin de asegurar el cumplimiento de los propósitos del Protocolo. En Chile esta medida no se ha llevado a cabo e implementarla en los entornos de las AMP permitiría alivianar el peso de la explotación en la "milla 201", lo que impactaría menos en los recursos vivos. Además, al estar en el marco CPPS, no implica un menoscabo el hecho de que tales zonas estén fuera de la jurisdicción nacional de alguno de los países miembro.

\section{La potencialidad de las AMP como laboratorios naturales}

La ciencia y tecnología son una arista transversal a la responsabilidad oceánica, al cumplir un rol importante en cómo se configuran los intereses marítimos nacionales. En dicha línea, el concepto de "laboratorio natural" surge como un foco al que Chile puede direccionar los esfuerzos en AMP, entendiéndolo como condiciones geográficas y características naturales únicas que transforman al país en un lugar privilegiado para la ciencia "y al mismo tiempo nos impone la responsabilidad frente al planeta de ser los 'guardianes' de estas riquezas y biodiversidad naturales (...) Chile debe enriquecer su identidad internacional, su Imagen País, a partir de esta condición única. Al mismo tiempo, estas condiciones nos permiten ser activos participantes en desafíos científico-tecnológicos globales" (MINREL, 2018, p. 81).

Puede apreciarse que tal concepto tiene la consciencia de la responsabilidad que le cabe, a la vez que la proyección internacional que entrega desde el área de la ciencia, a favor de Chile y sus intereses. Eso no es ajeno en temas oceánicos: la ley 18.892 incluye el enfoque precautorio (siendo el objetivo pasar de la precaución a la certeza) y la PON indica que "[e]I conocimiento científico y la información útil y oportuna sobre los océanos constituye la base para el diseño e implementación de políticas públicas y la toma de decisiones oportunas que aseguren la conservación de los ecosistemas marinos y el uso sustentable del océano para contribuir al bienestar social y la identidad cultural nacional" (CMDPO, 2018, p. 53).

Esto es funcional a las AMP, al ser zonas que, por su carácter de protección de las especies y ecosistemas, tienen una alta capacidad de atracción para la investigación científica, lo cual debe tener un vínculo con el bienestar social y el desarrollo local. En tal senda, 
figura el archipiélago de Juan Fernández como modelo global de conservación. Así, National Geographic Pristine Seas -equipo de investigadores y científicos-fue a reforzar el histórico proceso que la comunidad genera en pesca sostenible. Los resultados de su investigación determinaron que, de las especies de peces observadas en 2013 , el $96 \%$ eran endémicas y que, de las especies de peces observadas en 2017, el 99\% eran endémicas. El equipo presentó estos resultados de la investigación a los pescadores, gremio del turismo y la comunidad, producto de lo cual, ese mismo año, el archipiélago solicitó la expansión de la zona de pesca artesanal a 24 millas, de la milla 24 a la 200, un parque marino (Sala \& Muñoz, 2017, p. 87). Tal es el más fehaciente modelo de vinculación entre ciencia y comunidad en un AMP.

En el plano general, la realidad hace que el entendimiento entre las instituciones, la academia y las comunidades sea fundamental para el éxito. En esa dirección va la Iniciativa Milenio, un programa científico del Ministerio de Economía, que entre sus áreas cuenta con el Instituto Milenio de Oceanografía, y los Núcleos Milenio de Conservación Marina y de Ecología y Manejo Sustentable de las Islas Oceánicas. Con ello, apuntan a divulgar los descubrimientos y educar a los tomadores de decisiones y a la sociedad en su conjunto. Eso se complementa con la acción desde el MINREL, que impulsa una política exterior que aproveche el capital humano y los laboratorios naturales existentes. Para ello, busca posicionarse como catalizador y, velando por la incorporación de la dimensión internacional, facilitador del trabajo por medio de la conexión a redes globales, junto al regreso e integración laboral de los becarios, para una permanente interacción entre lo nacional-internacional, y apoyo de la internacionalización, poniendo la ciencia al servicio de la soberanía e inserción nacional (MINREL, 2018, p. 84).

El desafío es cómo coordinar esta iniciativa con los otros actores involucrados en las ciencias marinas, a través del reforzamiento de la institucionalidad existente en la materia, específicamente con el Comité Oceanográfico Nacional (CONA), que bajo la dirección del Servicio Hidrográfico y Oceanográfico de la Armada (SHOA) coordina varios participantes. El Comité ha creado dos documentos clave: el Plan Oceanográfico Nacional en 2010 y la Propuesta de Política Nacional en Investigación Científica Marina en 2005. Pero, aunque la fuente de 2005 se veía como la guía para la ciencia marina, no pasó del rango de propuesta.

Aquello dio pie a que el Plan recogiera aspectos postulados en la Propuesta de 2005. Como el señalamiento de líneas de investigación prioritarias para el desarrollo nacional, entre las que estaba la prospección de recursos vivos y no vivos, y el manejo de recursos marinos; y posicionar cuatro ejes que la investigación científico-marina requiere: recursos humanos, infraestructura, equipamiento y economía (CONA, 2010). Otra área clave son los 850 cupos para carreras universitarias en ciencias del mar, repartidos en ocho carreras y tres instituciones que dictan cursos de maestría o doctorado. Esto se complementa con que existen sólo tres publicaciones periódicas indexadas dedicadas a la ciencia y tecnología del mar. Este dato se proyecta a lo que la PON señala acerca de artículos científicos publicados por chilenos, indicando que en el periodo 2008-2016 fueron 449 relativos a investigación oceánica, habiendo en Chile, por cada millón de habitantes, diez investigadores en ciencias oceánicas (CMDPO, 2018, p. 55). 
Esto denota el grado de avance de las ciencias marinas, que debe ser potenciado en el futuro, para aumentar cualitativa y cuantitativamente los recursos humanos especializados en el área, y que esto derive en una mayor actividad investigativa con difusión en los medios competentes al área. Adherido a ello, resulta de interés ver la orientación de la formación en Chile para temas marinos. De los 850 cupos, 380 fueron para pesca y acuicultura, mientras que 407 fueron para oceanografía, biología marina y química marina. Esto muestra lo que podría entenderse como un equilibrio entre la visión economicista y la conservacionista.

A la par, un valioso aporte del CONA es el paneo a las fuentes de financiamiento para la investigación marina. Identifica siete fondos estatales, que significaron $\$ 3.160$ millones, que se dividieron en 48 proyectos (cifra que debe actualizarse) (CONA, 2010, p. 17). Un monto que podría ser mayor, pero ello debe contextualizarse en una realidad nacional donde se invierte apenas $0,39 \%$ en investigación y desarrollo, por lo que el desafío es general.

Ahora bien, la concesión de este panorama respecto de la ciencia marina, visto desde las AMP como espacios para la realización de actividades de un Laboratorio Natural, debe estar dado en ciertas acciones que demuestren ser un aporte al desarrollo regional y local, y que deben contar con el MINREL como partícipe. Allí se centra su rol de articulador nacional e internacional de la PON: para responder a la bidireccionalidad de la diplomacia (Laborda, 2018) y para que la captación de capacidades, conocimientos y habilidades vinculadas a la ciencia y tecnología en temas oceánicos sea la piedra que refuerce a Chile como laboratorio natural.

En particular, áreas como los parques marinos, restringidas para todo tipo de pesca, son un inmejorable laboratorio para la investigación de la biodiversidad, lo cual da pie a que se potencien disciplinas tanto biológicas como químicas y geológicas. Esto puede derivar en la prospección (como planteaba el CONA) y descubrimiento de recursos genéticos nuevos, lo que conlleva una intensa labor de reconocimiento de origen -uno de los temas de futuro, como lo reflejan las mesas de trabajo del BBNJ. Esto implica una diplomacia capaz de posicionar los intereses nacionales ante los distintos foros y organismos internacionales.

Por otra parte, una cooperación de la tecnología hacia zonas tan vastas como las AMP en las islas oceánicas, se demuestra en la subscripción del Memorándum de Entendimiento en 2016 entre Chile y Reino Unido, sobre cooperación en materia de aplicaciones satelitales. Ello permitiría contar con el sistema Catapult para la entrega de imágenes satelitales sobre naves pesqueras que evidencien actividades de pesca INDNR (Aimone, 2016); o las ONGs que han ofrecido financiamiento para el monitoreo de sus AMP. Ambas alternativas denotan que, en cuanto a la protección oceánica, las alianzas sobrepasan el marco convencional, ampliándose hacia vínculos entre actores estatales y no-estatales, lo que ratifica que la acción de la responsabilidad oceánica es intersectorial y a distintas escalas en su compromiso.

Mientras para en los parques marinos se vislumbra esta situación, para las AMCP-MU, que permiten la pesca artesanal, la ciencia y tecnología tiene otros roles en el cumplimiento de la responsabilidad oceánica. La entrega de las certezas científicas e información es un factor de preponderancia para que las comunidades locales generen un manejo sostenible 
de los recursos; eso es lo que ocurrió en los Bosques de Calabacillo de Navidad: el AMP fue circundada por las parcelas de cochayuyo, buscando un equilibrio económico-ambiental.

En dicho sentido, la investigación científica puede colaborar a que se replique en otras partes de Chile el ejemplo de Juan Fernández, donde sus pescadores lograron que la langosta y el cangrejo dorado obtuviesen el sello de origen e indicación geográfica en Chile y fuesen certificados por el Marine Stewardship Council como una pesca sostenible. Este tipo de iniciativas apunta directamente hacia el desarrollo económico regional y local, pues da un valor agregado a tales productos para su venta. Otras comunidades pueden aspirar a tales distinciones, o incluso otras, como hacer que sus productos posean certificado de trazabilidad para que los compradores sepan que están consumiendo un bien artesanal, sostenible y que ha sido llevado a su mesa por medio de una acción tendiente a la responsabilidad oceánica.

El involucramiento de actores como PROCHILE en estos procesos resulta positivo, pues son un órgano del MINREL con presencia regional, que puede contribuir a que este tipo de bienes, productos de la aplicación de conocimientos científicos y tecnológicos a temas locales, sean internacionalizados. A la vez, esto es beneficioso para las comunidades en relación con el valor agregado de sus exportaciones, lo es también para Chile, en dar la imagen de país responsable en temas oceánicos, mediante un uso sostenible de los recursos. Aquello compete a la diplomacia, porque la captación de cooperación técnica o la atracción de nuevas investigaciones marinas, promoviendo al país como Laboratorio Natural, está en sus manos. En complemento, la visibilización de productos con sello de origen y/o certificados de pesca sostenible va asociada al nexo local-global que es bidireccional y el MINREL debe encausar.

\section{Conclusiones y desafíos}

A lo largo de esta investigación se ha analizado la gestión de las Áreas Marinas Protegidas como un ejercicio de la responsabilidad oceánica, como forma de aproximarse al tema, desde la política exterior, en dirección hacia el desarrollo nacional. Es así como la responsabilidad oceánica se ha propuesto como un principio activo, un compromiso en la acción para el uso sostenible de los recursos y ecosistemas marinos, pero interpretado desde lo político para fortalecer las capacidades de implementación y construir la legitimidad que los procesos bottom-up propician. Asimismo, al ser transversal, puede aunar actores involucrados en el interés superior de obtener océanos sostenibles.

De ese modo, la responsabilidad oceánica complementa otras áreas, como el interés en el tema económico, pues existe una ligazón entre el crecimiento económico y la sostenibilidad oceánica. Por eso, la armonización entre conservacionismo y economía es fundamental para el éxito de una Política Oceánica Nacional.

Fundado en estas consideraciones, pueden plantearse cinco conclusiones y/o nuevas líneas de investigación a futuro para los desafíos que el país deberá enfrentar en temas oceánicos y que pueden afrontarse de mejor forma mediante el ejercicio de una responsabilidad oceánica. En primer lugar, se develó el estado de situación de aquel 42\% de ZEE declarada AMP, lo que evidenció 29 planes de administración faltantes, a los cuales ha de darse 
seguimiento -en caso que, a partir de las recomendaciones que la OCDE entrega para estas áreas, se proyecta el compromiso político actual de Chile.

En segundo lugar, se presentó cómo la institucionalidad chilena ha propiciado un fortalecimiento para dar viabilidad a la protección de las AMP, ejemplificado en la SBAP o la intención de darle carácter permanente al Consejo de Ministros que creó la PON. Sin embargo, se aprecia que aquello debe aparejarse a los recursos humanos y financieros para operativizar las AMP, superando una posible fragmentación en la coordinación.

En tercer lugar, este estudio deja abierta la puerta a futuras investigaciones del área de políticas públicas y de la ciencia y tecnología, que examinen la masa crítica que existe en el país en la materia, de cara a la real posibilidad de consolidar a Chile y sus AMP como laboratorios naturales de talla mundial, teniendo en consideración tanto el capital humano altamente calificado como los fondos destinados por el Estado para ello, pues sirve como información base para las políticas públicas en la materia.

En cuarto lugar, sobre desarrollo regional y local, se concluye que una política nacional para temas oceánicos, aunque es un avance significativo, no es capaz de reflejar y dar una bajada concreta a las diversas realidades de Chile en la relación con el océano, como lo haría un desagregado de "Políticas Oceánicas Regionales" que complemente. Esto se relaciona con los otros tópicos, pues la ciencia puede entregar herramientas al servicio de lo local y vincularlo a lo global. En tal forma, el estudio de casos exitosos como el modelo creado en Juan Fernández, aquí presentado, es útil como insumo para la reflexión acerca de gobernanza local en AMP y elmanejo sostenible de recursos. Además, considerando que el enfoque de este escrito es la política exterior, se deja abierta la puerta a estudios en la materia, pero desde el ámbito del comercio internacional y cómo el MINREL puede apoyar la internacionalización de productos locales con sellos de sostenibilidad y/o trazabilidad para fomentar una economía de mayor valor agregado.

Finalmente, en quinto lugar, ha quedado evidenciada la importancia de la política exterior y el rol de Cancillería como el articulador de la PON, clave para el ejercicio de la responsabilidad oceánica. El aprovechamiento de la visibilización que obtuvo Chile gracias a su acción por las AMP puede hacer que Chile pase de referente en la declaración de estas, a ser un referente amplio en temas oceánicos, asumiendo la responsabilidad por cumplir. Desde un rol cooperativo, a la vez que recibiendo recomendaciones de otros actores (no solo Estados), el país puede buscar buenas prácticas en otros lugares, posibles de replicar. O concertar cooperación en temas como la tecnología, que es una herramienta para el desarrollo en las AMP, pues los sistemas satelitales como el Catapult reforzarían las labores de monitoreo, tan difíciles en áreas como las AMP en las islas oceánicas.

Por otra parte, hay temas de futuro que se avecinan en lo multilateral y Chile tendrá que tener una postura clara y propositiva para tales desafíos. La inminente negociación del BBNJ es un nuevo frente que se abre. Temas como las AMP fuera de la jurisdicción nacional o cuestiones relativas a recursos biológicos y genéticos marinos serán ampliamente debatidos y Chile ha de buscar resguardar y maximizar sus intereses, a la vez que saber coordinarse con otros países de posturassimilares, fortaleciendo su vocación oceánica. 


\section{Referencias}

Acuerdo sobre la aplicación de las disposiciones de la Convención de las Naciones Unidas sobre el Derecho del Mar del 10 de diciembre de 1982 relativas a la conservación y ordenación de las poblaciones de peces transzonales y las poblaciones de peces altamente migratorios. 1994.

Aimone, G. (2016). Sustentabilidad del Océano: combate de la pesca ilegal y los parques marinos. Revista de Marina, 960, 14-21.

Araos, F. \& Ther, F. (2017). How to adopt an inclusive development for marine conservation: preliminary insights from Chile. Current Opinion in Environmental Sustainability, 24, 68-72.

Cañas, R. (1954). El Pacífico, epicentro geopolítico de un nuevo mundo en estructuración. Terra Australis, 12, 11-16.

Comisión Permanente del Pacífico Sur. (1989). Protocolo para la Conservación y Administración de las Áreas Marinas y Costeras Protegidas del Pacífico Sudeste. CPPS.

Comisión Permanente del Pacífico Sur. (1952). Declaración sobre Zona Marítima. CPPS.

Comité Oceanográfico Nacional. (2010). Plan Oceanográfico Nacional. Servicio Hidrográfico y Oceanográfico de la Armada.

Consejo de Ministros para el Desarrollo de la Política Oceánica Nacional de Chile. (2018). Política Oceánica Nacional de Chile.

Convención de las Naciones Unidas sobre el Derecho del Mar. 1982.

Convención sobre la Conservación y Ordenamiento de los Recursos Pesqueros en Alta Mar en el Océano Pacífico Sur. 2009.

Convenio sobre la Diversidad Biológica. 1992.

Colacrai, M. \& Lorenzini, M. (2005). Política exterior de Chile: ¿Excepcionalidad o continuidad? Una lectura combinada de fuerzas profundas y tendencias. CONFines de Relaciones Internacionales y Ciencia Política, 1 (2), 45-63.

Decreto 238 de 2005. Reglamento sobre parques marinos y reservas marinas de la Ley General de Pesca y Acuicultura.

Dictamen 077778N13 de 2013 [Contraloría General de la República]. Respecto de la categoría Áreas Marinas y Costeras Protegidas de Múltiples Usos, y su relación con el Sistema Nacional de Áreas Silvestres Protegidas del Estado, en particular en relación con el artículo 158 de la Ley General de Pesca y Acuicultura.

Durosselle, J. (2000). Todo imperio perecerá. Teoría sobre las relaciones internacionales.

Fondo de Cultura Económica.

Dussel, E. (2011). Filosofía de la Liberación. Fondo de Cultura Económica.

González, G. (1947). Declaración Oficial del Presidencia de Chile 23 de junio.

Jaguaribe, H. (1974). Brasil: crisis y alternativas. Amorrortu.

84 Relaciones Internacionales Vol 29 - № 59/2020. 
Laborda, C. (2018). Diplomacia Oceánica y Conservación de la Biodiversidad. En J. Somavía \& P. Oyarce (Eds), Chile, Actor del Sistema Multilateral. Una Tradición Nacional (pp. 537-584). Academia Diplomática de Chile-SEGIB.

Ley 20657 de 2013. Modifica en el ámbito de la sustentabilidad de recursos hidrobiológicos, acceso a la actividad pesquera industrial y artesanal y regulaciones para la investigación y fiscalización, la ley general de pesca y acuicultura contenida en la ley 18.892 y sus modificaciones.

Ley 20417 de 2010. Crea el Ministerio, el Servicio de Evaluación Ambiental y la Superintendencia del Medio Ambiente.

Ley 20249 de 2008. Crea el Espacio Marino Costero de los Pueblos Originarios.

Ley 19300 de 1994. Ley sobre Bases Generales del Medio Ambiente.

Ley 18892 de 1991. Ley General de Pesca y Acuicultura.

Martínez, J. (1994). Política Oceánica Nacional, sugerencias para una formulación, Texto de la clase magistral dictada en el Teatro Municipal del Viña del Mar.

Ministerio de Relaciones Exteriores de Chile. (2018). Política Exterior de Chile 2030. MINREL.

National Geographic Pristine Seas, Waitt Foundation, Oceania \& Núcleo Milenio de Ecología y Manejo sustentable de las Islas Oceánicas. (2017). Archipiélago de Juan Fernández: biodiversidad marina y necesidades de conservación. Informe científico.

Organización para la Cooperación y el Desarrollo Económico. (2017). Marine Protected Areas. Economics, Management and Effective Policy Mixes.

Organización para la Cooperación y el Desarrollo Económico \& Comisión Económica para América Latina y el Caribe. (2016). Evaluaciones del Desempeño Ambiental Chile 2016.

Ortega, R. (2015). La Política Marítima. En M. Artaza \& C. Ross. La Política Exterior de Chile, 1990-2009 (pp. 166-189). RIL Editores.

Our Ocean Chile. (2015). Our Ocean 2015 Initiatives.

Programa de las Naciones Unidas para el Medio Ambiente. (2010). El Plan Estratégico para la Diversidad Biológica 2011-2020 y las Metas de Aichi para la Diversidad Biológica.

Sala, E. \& Muñoz, A. (2017). Valor para proteger el futuro. National Geographic en español, octubre, 70-89.

Subsecretaría de Pesca y Acuicultura. (2018). Estado de Situación de las Principales Pesquerías Chilenas, año 2017.

Zegers, F. (2018). Chile y la Negociación de la Convención de Naciones Unidas sobre el Derecho del Mar. En J. Somavía \& P. Oyarce (Eds). Chile, Actor del Sistema Multilateral. Una Tradición Nacional (pp. 597-634). Academia Diplomática de ChileSEGIB. 


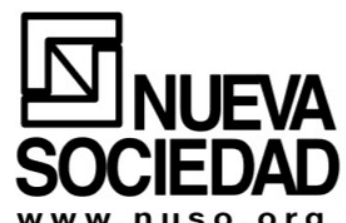

Directora: Svenja Blanke Jefe de redacción: Pablo Stefanoni
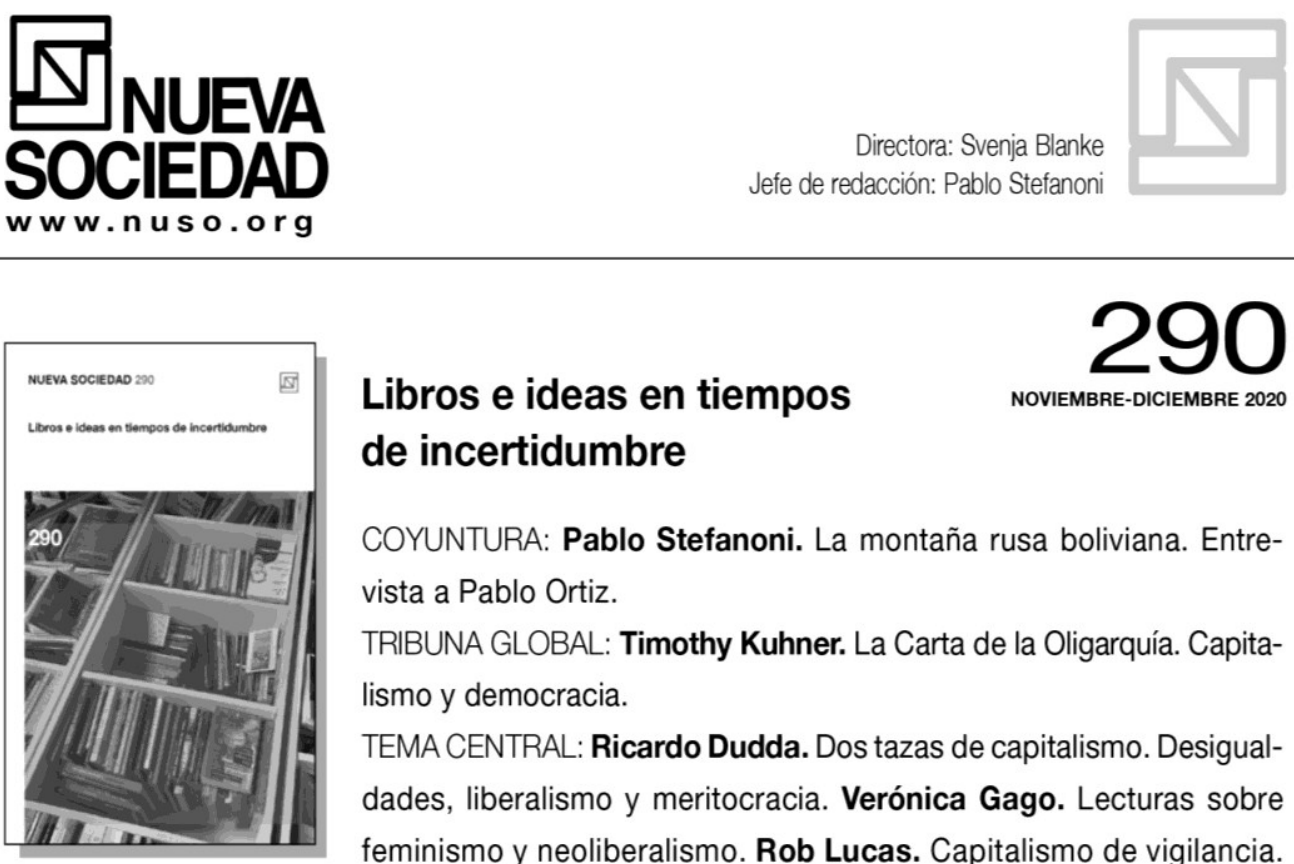

\section{Libros e ideas en tiempos de incertidumbre}

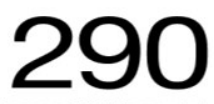

NOVIEMBRE-DICIEMBRE 2020

COYUNTURA: Pablo Stefanoni. La montaña rusa boliviana. Entrevista a Pablo Ortiz.

TRIBUNA GLOBAL: Timothy Kuhner. La Carta de la Oligarquía. Capitalismo y democracia.

TEMA CENTRAL: Ricardo Dudda. Dos tazas de capitalismo. Desigualdades, liberalismo y meritocracia. Verónica Gago. Lecturas sobre feminismo y neoliberalismo. Rob Lucas. Capitalismo de vigilancia.

Yanina Welp. La democracia y el declive de las elites. Marc Saint-Upéry. Red Mirror: ¿qué futuro se escribe en China? Entrevista a Simone Pieranni. Benjamin Kunkel. ¿De la socialdemocracia al socialismo? Ingrid Spiller. ¿Cómo cambiar el mundo? Pablo Carmona Pascual. Patriotas indignados, Europa como fantasma. Tomás Borovinsky. Fragmentar el futuro. Hacia una nueva relación humano/no humano. Juan Duchesne-Winter. ¿Por qué el comunismo resulta «insoportable»? Más allá de la economía libidinal.

ENSAYO: Eduardo Grüner. Sartre: hacia una moral de la ambigüedad.
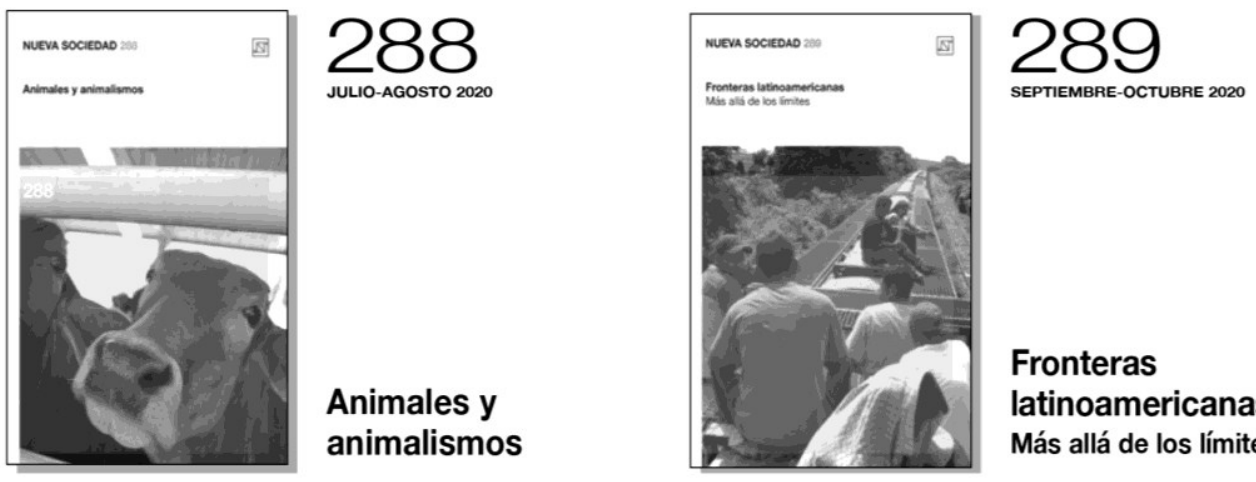

SEPTIEMBRE-OCTUBRE 2020

\section{Fronteras} latinoamericanas Más allá de los límites

PAGOS: Solicite precios de suscripción y datos para el pago a <info@nuso.org> $0<$ distribucion@nuso.org>.
EN NUESTRO PRÓXIMO NÚMERO

Geopolíticas latinoamericanas 